\title{
Fatigue analysis for the first row of blades of a micro turbine under full loading cycle using transient modal analysis
}

\author{
Ali Soleimani ${ }^{*}$, Saeid Shahraki ${ }^{\mathrm{b}}$ and Hosein Bisadi ${ }^{\mathrm{a}}$
}

${ }^{a}$ Iran University of Science and Technology, Narmak, Tehran, Iran

${ }^{b}$ School of Mechanical Engineering, University of Zabol, Iran

A R T ICLE IN F O ABSTRACT

\begin{tabular}{l}
\hline Article history: \\
Received 6 January, 2015 \\
Accepted 20 May 2015 \\
Available online \\
22 May 2015 \\
\hline Keywords: \\
First row of blades \\
Micro turbine \\
Full loading cycle
\end{tabular}

\section{Introduction}

One of the most major issues concerning warplanes during their flight time is the turbine blades' failure due to creeping-fatigue effect. When the blades at unusual high temperatures are put under high stress, they suffer from creeping effect. At first surface micro cracks are developed due to the slip of boundary grains. W-shape cracks are created due to the presence of carbide and as well as high amounts of stress, which in times cause fatigue cracks to germinate. Consequently, the blade fails under low fatigue cycle (Salam et al., 2002). Chen et al. (2007) predicted the turbine blade's lifetime under creeping and fatigue effects using modified linear damage summation (MLDS) and Modified strain range partitioning (MSRP) methods. They first performed an accurate analysis via finite element method (FEM) considering the maximum thermal load, centrifugal force, and the force caused by airflow, to evaluate the blade's lifetime. After that, they analyzed the stress-strain response of the lowcycle fatigue (LCF) as well as creeping-fatigue effect at constant temperature state, plastic hardening,

* Corresponding author.

E-mail addresses: alisoleimani@rail.iust.ac.ir (A. Soleimani)

(C) 2015 Growing Science Ltd. All rights reserved.

doi: $10.5267 /$ j.esm.2015.5.004
In advanced modern turbines, the temperature of the turbine's first blade row which is called the turbine's hot part, increases almost as $1000^{\circ} \mathrm{C}$. These blades must operate for long periods of time. Since, the main failure reason of the parts operating under high temperature and cyclic
loading conditions is fatigue as well as creeping phenomena, acquiring an accurate estimate of these blades' lifetime under creeping and fatigue interactions is very necessary for theoretical and practical requirements. The fatigue of the first blade row of the micro turbine TRI60 is heat transfer of blades as well as thermal stress is analyzed at first via ABAQUS software. Consequently, a fatigue analysis under full cycle and within the interval of $\frac{2}{3} v_{\max }$ to $v_{\max }$ is software.

(C) 2015 Growing Science Ltd. All rights reserved. 
and considering the contact of lower blade parts (the root) with other parts. Kim et al. (2008) analyzed the failure of J85 turbine blade. They concluded the following results after analyzing the required data from fractography analysis, metallography, and blade coatings:

- The damages induced on outer parts of motor are secondary damages which are caused by motor vibrations due to the failure of the turbine's rotor first blade row.

- Since the blades of the first row are subjected to superheated air, they suffer from creeping failure.

- Development of the creeping speed in failed blades is because of the blades being subjected to superheated air for more than 6 times before.

- An accurate measurement of the length as well as the thickness of the blade is necessary for the purpose of preventing the blade from creeping effect.

Yan et al. (2014), Yan and Nie (2008) investigated the vibrational characteristics of the materials' fatigue effect at different turbine parts. For this purpose, they first designated critical areas of the blade and then analyzed these areas by performing experimental tests. In this paper, following a brief description of loads applied to the blades, fatigue of first blade row of a micro turbine is analyzed numerically.

\section{Mechanical loads developed in the blade}

\section{1 axial loads}

For static strength analysis, only the amount of mean airflow force is considered and its distribution is assumed to be linear. This force is applied on the front and back parts of the blade in the form of pressure difference, which is neglected due to its small value, according to (Chen et al., 2005).

\subsection{Thermal loads}

There has always been a direct relation between the developments and progresses in air industry and increasing the turbine's inlet temperature. This temperature is of particular importance for the blades. Therefore, thermal analysis of the blades has always been significantly important. Modern industrial materials such as single-crystal nickel-based super alloys and blade cooling structures such as inside-blade cavities are important for turbine blades, which are not useful for micro turbines due to small available space. On the other hand, according to some studies, cooling cases along with thermal coatings can lower the blade's surface temperature as one-thirds of the initial value (Yang et al., 2014). To acquire a prediction of the fatigue and creep lifetime, an accurate estimate of the temperature is required, since the temperature is a major factor in fatigue effect at high temperatures. For instance, if temperature increases from 32 to $800^{\circ} \mathrm{C}$ the low cycle fatigue (LCF) lifetime decreases as $23 \%$ (Chen $\&$ Xie, 2005). Therefore, taking the temperature distribution effect into consideration is necessary for structural strength prediction. Hence, temperature distribution of blade containing thermal coatings is investigated in this research.

\subsection{Centrifugal force}

Centrifugal force, which is one of the major reasons for creeping and fatigue effects in turbine blades, is considered as one of the most unwanted loadings on turbine rotary parts. This force depends on variable parameters, including rotational velocity of rotor, the distance of elements from rotating axis, and the material. Centrifugal force has the largest value among applied forces on the turbine's blade and the significant size of this force causes the grains to move at high temperatures. This can cause deformation of the blade that is called creeping effect which can lead to failure. Here, the maximum operating revolution of TRI60 micro turbine is $30000 \mathrm{rpm}$. 


\section{Finite element model of the blade}

The first blade row of turbine along with the disk is modeled via Solidworks software (Fig. 1).

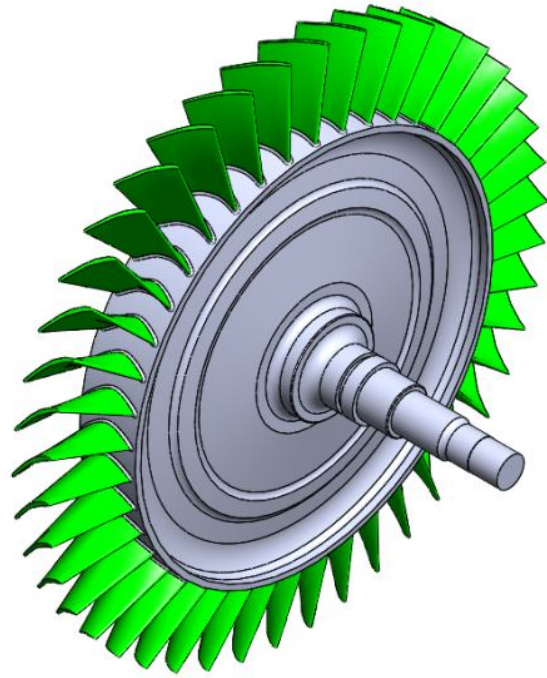

Fig. 1.3D model of the blade and the disk

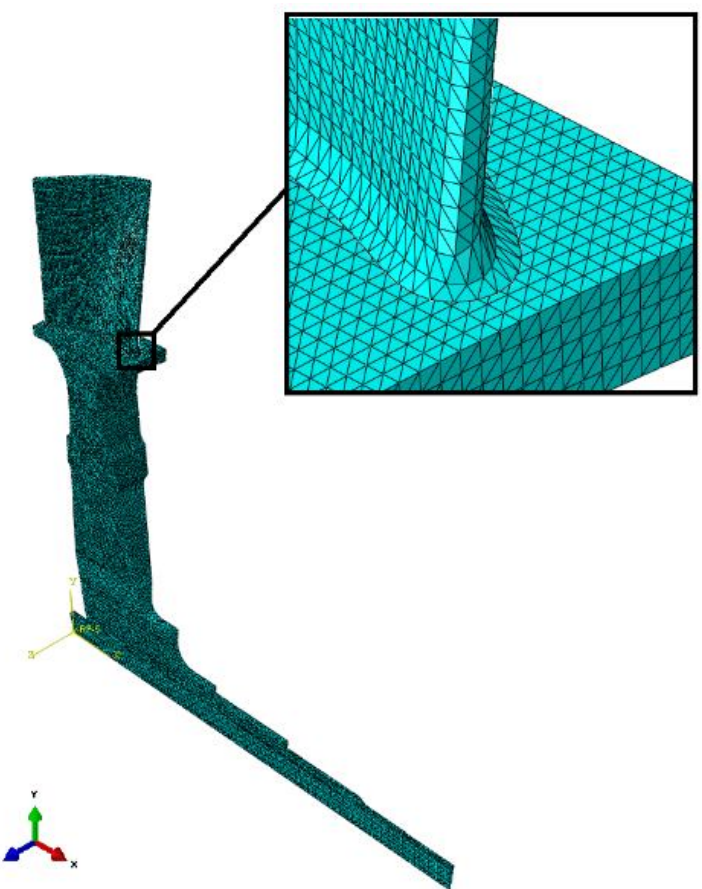

Fig. 2. Finite element model of the first row blade section ( $1 / 43$ of the total turbine model)

This model includes 43 blades mounted on the disk. Due to similarity of the blades, only one blade is analyzed. Fig. 2 shows finite element model of this blade. This model consists of 99391 tetra elements. The blades are made from In718 super alloy, which is assumed to be the forming material of the disk as well. Mechanical characteristics of this material are listed in Table 1.

\section{Table 1}

Mechanical characteristics of In718 super alloy (Metals, 2007)

\begin{tabular}{|c|c|c|c|c|c|}
\hline Temperature $\left({ }^{\circ} \mathrm{C}\right)$ & $\mathrm{E}(\mathrm{GPa})$ & $\begin{array}{c}\text { Poisson } \\
\text { Ratio } \\
\end{array}$ & Density $(\mathrm{kg} / \mathrm{m} 3)$ & $\begin{array}{c}\text { Mean coefficient of } \\
\text { thermal expansion }(\mathrm{e}-6) \mathrm{K}\end{array}$ & $\begin{array}{c}\text { thermal } \\
\text { conductivity }(\mathrm{W} / \mathrm{m} \mathrm{K}) \\
\end{array}$ \\
\hline 21.11 & 199.926 & 0.294 & 8220 & - & 11.0981204 \\
\hline 37.77 & 198.5472 & 0.291 & 8220 & 13.158 & - \\
\hline 93.33 & 195.7896 & 0.288 & 8220 & - & 12.39530331 \\
\hline 148.88 & 193.032 & 0.28 & 8220 & 13.554 & - \\
\hline 204.44 & 190.2744 & 0.28 & 8220 & - & 14.12488051 \\
\hline 260 & 186.8274 & 0.275 & 8220 & 13.932 & - \\
\hline 315.55 & 184.0698 & 0.272 & 8220 & - & 15.99858915 \\
\hline 371.11 & 180.6228 & 0.273 & 8220 & 14.346 & - \\
\hline 426.66 & 177.8652 & 0.271 & 8220 & - & 17.72816636 \\
\hline 482.22 & 174.4182 & 0.272 & 8220 & 14.562 & - \\
\hline 537.77 & 170.9712 & 0.271 & 8220 & - & 19.45774356 \\
\hline 593.33 & 166.8348 & 0.276 & 8220 & 15.102 & - \\
\hline 648.88 & 163.3878 & 0.283 & 8220 & - & 172.9577206 \\
\hline 704.44 & 158.562 & 0.292 & 8220 & 16.038 & - \\
\hline 760 & 153.7362 & 0.306 & 8220 & - & 23.06102941 \\
\hline 815.55 & 146.8422 & 0.321 & 8220 & - & - \\
\hline 871.11 & 139.2588 & 0.331 & 8220 & - & 24.93473805 \\
\hline 926.66 & 129.6072 & 0.334 & 8220 & - & - \\
\hline 982.22 & 119.9556 & 0.341 & 8220 & - & 26.66431525 \\
\hline 1037.77 & 109.6146 & 0.366 & 8220 & - & - \\
\hline 1093.33 & 98.5842 & 0.402 & 8220 & - & 28.24976102 \\
\hline
\end{tabular}




\section{Thermal analysis of blade}

When temperature variations and heat transfer coefficient come together, thermal stress develops. Noting that micro turbine blades do not have cooling cases and heat transfers along them with a high speed, the blade finds the turbine's inlet temperature in a short time and consequently, there is no thermal stress in these blades. For the purpose of ensuring the temperature of the whole part, transient thermal analysis is performed according to Yang et al. (2014).

For blade coatings, mostly NiCrAlY bonds and ZrO2-8 wt\% Y2O3 ceramic coatings are used. Usually, the thickness of bonds and ceramic coatings equals $100 \mu \mathrm{m}$ and $300 \mu \mathrm{m}$, respectively, and the thermal grown oxide part (TGO) is subjected to oxidation at high temperatures and its maximum thickness is taken within $10-50 \mu \mathrm{m}$ in the literature, which is assumed to be $50 \mu \mathrm{m}$ in this study. The characteristics of this section, including structure, compounds, and roughness, are not coated uniformly. It is shown experimentally that the influence of the thickness of this part on stress is negligible (Wang $\&$ Xiao, 2004). Mechanical as well as thermal characteristics of each of these coatings are listed in Table 2. Fig. 3 shows the finite element model of the blade with thermal coatings, which consists of ceramic coating (top bond coat, TBC), thermal grown oxide (TGO), and bond coat (BC), as mentioned before.

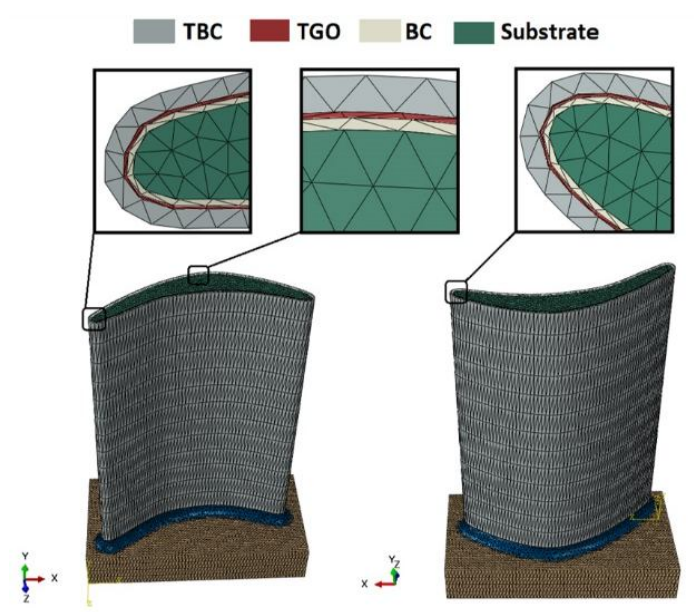

Fig. 3. Finite element model of the blade with thermal coatings

In this analysis, the initial temperature of the whole part and the temperature of blade surface are assumed to be $20^{\circ} \mathrm{C}$ and $1000^{\circ} \mathrm{C}$, respectively. Fig. 4 shows the acquired results from transient thermal analysis. Figs. $4 \mathrm{a}$ and $4 \mathrm{~b}$ show the blade's temperature distribution during 2 and 4.5 seconds from the beginning, respectively. As it is seen from the figures, the temperature of the intermediate part of the blade remains $20^{\circ} \mathrm{C}$ during the first 2 seconds of the analysis, and after 4.5 seconds, the whole temperature of the blade becomes $1000^{\circ} \mathrm{C}$.

Table 2

Mechanical and thermal characteristics of the blade and blade coatings

\begin{tabular}{|c|c|c|c|c|c|c|c|c|}
\hline & $\begin{array}{l}\text { Temperature } \\
\text { range }(C)\end{array}$ & $\begin{array}{c}\text { Young's } \\
\text { modulus } \\
(\mathrm{GPa})\end{array}$ & $\begin{array}{l}\text { Poisson's } \\
\text { ratio }\end{array}$ & $\begin{array}{c}\text { Thermal } \\
\text { expansion } \\
\left(10^{6} / \mathrm{C}\right.\end{array}$ & $\begin{array}{c}\text { Yield } \\
\text { strength } \\
(\mathrm{MPa})\end{array}$ & $\begin{array}{c}\text { Thermal } \\
\text { conductivity } \\
(\mathrm{W} /(\mathrm{cm} \mathrm{K}))\end{array}$ & $\begin{array}{c}\text { Specific heat } \\
(\mathrm{J} /(\mathrm{kgK}))\end{array}$ & $\begin{array}{l}\text { Density } \\
\left(\mathrm{kg} / \mathrm{m}^{3}\right)\end{array}$ \\
\hline Substrate & $20-1093$ & $200-99$ & $0.294-0.402$ & $13.158-18$ & $1108-871$ & $0.11-0.282$ & $440-710$ & 8220 \\
\hline Bond coat & $20-1600$ & $200-110$ & $0.30-0.33$ & $13.6-17.6$ & $426 \rightarrow 114$ & $0.058 \rightarrow 0.170$ & 450 & 7380 \\
\hline TGO & $20-1600$ & $400-320$ & $0.23-0.25$ & $8.0-9.6$ & $\begin{array}{c}10,000(20- \\
\left.900^{\circ} \mathrm{C}\right)-1000\end{array}$ & $0.10 \rightarrow 0.04$ & 750 & 3984 \\
\hline Top coat & $20-1600$ & $48-22$ & $0.10-0.12$ & $9.0-12.2$ & - & $0.02 \rightarrow 0.017$ & 505 & 3610 \\
\hline
\end{tabular}




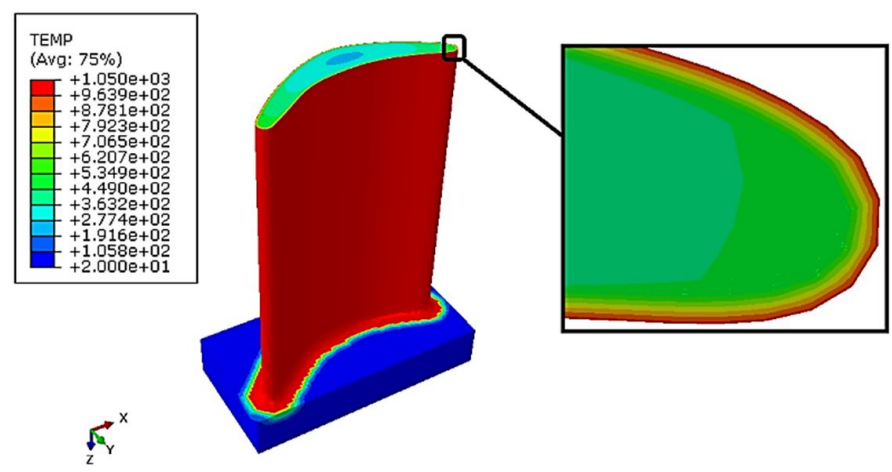

(a)

Fig. 4. Blade's thermal distribution in the presence of thermal covers, a) after 2 seconds, b) after 4.5 seconds

\section{Blade's strength analysis}

In this section, the weak points of the structure are located first. As mentioned before, the mean value of the airflow force is often assumed to be linear. This force exerts on the front and back parts of the blade as pressure difference and since its influence is negligible comparing with the centrifugal force, it is neglected. Boundary conditions are as follows:

- Circular symmetry conditions at two side parts as well as displacement constraint on the nodes of these two parts were applied to prevent convergence errors.

- Displacement constraints are applied on lower parts of the disk in radial and tangential directions, respectively.

- The rotational speed of the blade around the disk center is taken to be $30000 \mathrm{rpm}(3141.59$ $\mathrm{rad} / \mathrm{s})$.

Fig. 5 shows the blade's von-mises stress at temperature of $1000{ }^{\circ} \mathrm{C}$ as well as the maximum centrifugal force. As is shown in the figure, the maximum stress occurs at the middle of the blade at the region of the blade and disk contact, which equals to $620 \mathrm{MPa}$.

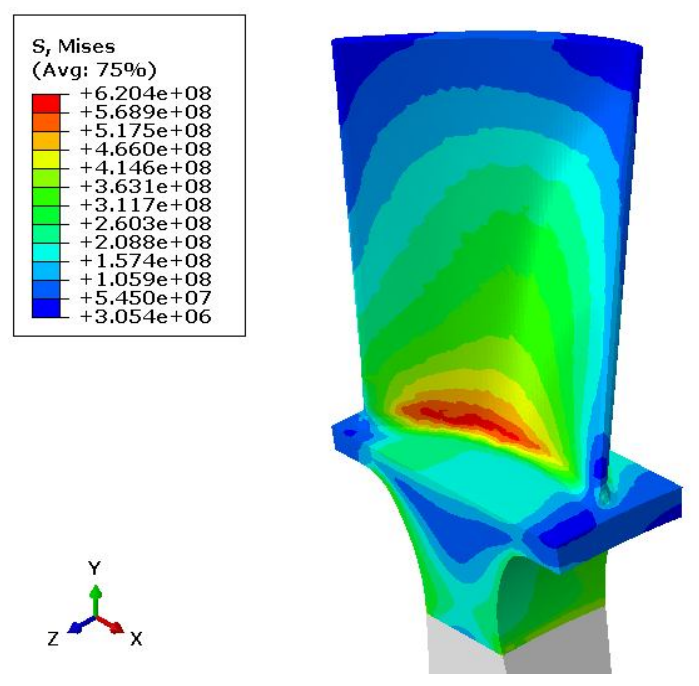

Fig. 5. Von-mises stress distribution due to centrifugal force. 
Fig. 6 shows the cross section of blade for the maximum von-mises stress due to centrifugal force. According to Fig. 6 and Fig. 7 it could be claimed that the areas which are under maximum stress have a $1 \mathrm{~mm}$ depth which can increase the risk of developing and growing cracks in these areas.

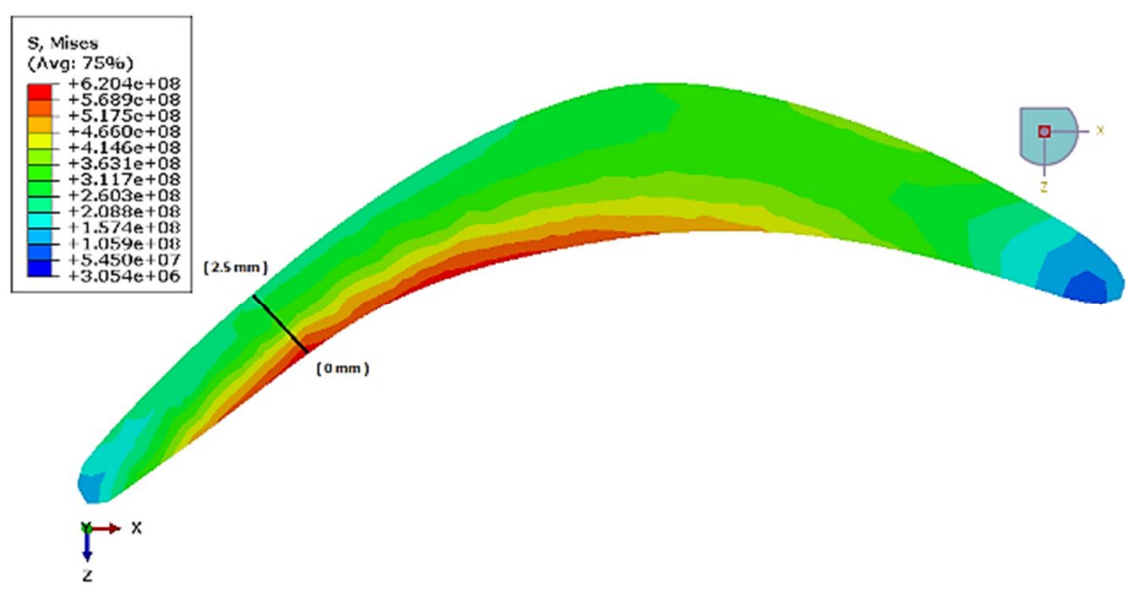

Fig. 6. Cross section of the maximum von-mises stress due to centrifugal force

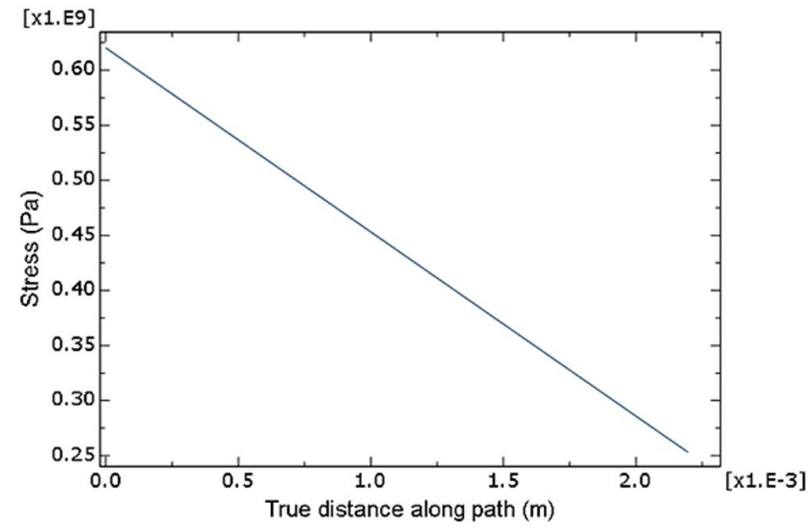

Fig. 7. The diagram of the maximum von-mises stress versus blade's width

Fig. 8 shows the von-mises stress versus temperature and velocity. In this analysis, combustion chamber temperature enhancement is assumed to change linearly with the increasing of the turbine's linear velocity. As shown in this figure, the maximum stress occurs at temperature of $1000{ }^{\circ} \mathrm{C}$, which equals the maximum stress due to centrifugal force in elastic region. Therefore according to the yield strength diagram of 718 super alloy shown in Fig. 9 the blade dose not enter into the plastic region.

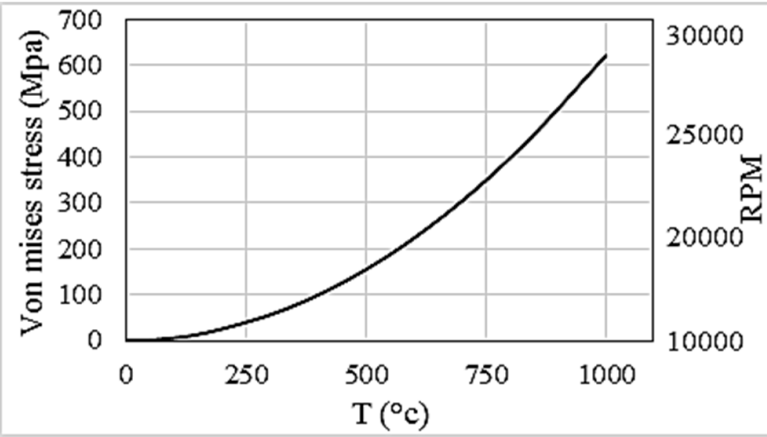

Fig. 8. Von-mises stress diagram versus temperature and turbine velocity

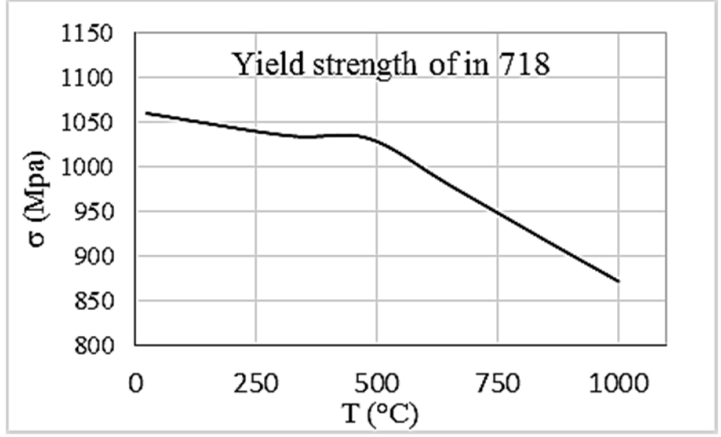

Fig. 9. Yield strength of 718 super alloy at various temperatures (International, 2002) 


\section{Fatigue analysis of the full cycle loading based on transient modal analysis}

When the motor reaches its maximum available revolution, the blade's temperature reaches $1000{ }^{\circ} \mathrm{C}$ and its mechanical stress reaches $620 \mathrm{MPa}$. In this section, the dynamic analysis of the blade is performed based on a 10 vibrational mode base excitation. Base excitations consist of vertical displacements in y direction caused by centrifugal force. This excitation is exerted in a time of 1 second. The transient modal finite element analysis is performed in two steps via ABAQUS software. At first, natural frequencies (natural modes) are analyzed. Then the blade displacements based on the considered excitation are acquired and finally, the fatigue analysis of the blade is performed via fe-safe software. Fatigue loading is such that the blade is put under the conditions of ambient temperature and no loading is applied at first and then it is put under the conditions of $1000{ }^{\circ} \mathrm{C}$ temperature and maximum stress (Fig. 10).

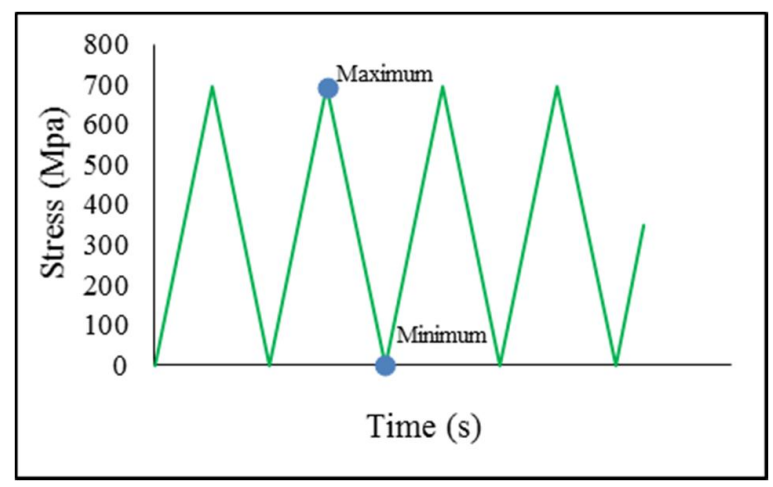

Fig. 10. The diagram of fatigue loading.

This fatigue loading is modeled by the blade displacement under the effect of centrifugal force as well as the first ten natural frequencies of the blade. Fig. 11 shows the first four natural frequencies of the blade, in which the first mode shape is happened at the frequency of $1839.8 \mathrm{~Hz}$. As it is shown in the figure, the first frequency is bending around $x$ axis, the second and third frequencies are torsion around $\mathrm{y}$ and $\mathrm{z}$ axes and the fourth frequency is bending around $\mathrm{x}$ axis. Also, Table 3 presents the first ten frequencies of the blade. The critical depreciation coefficient of the structure is considered as 0.0009 . This value is acquired by try and error. This coefficient causes the displacement of the whole model to be fixed. The larger this coefficient gets, the faster the displacement becomes a constant value.

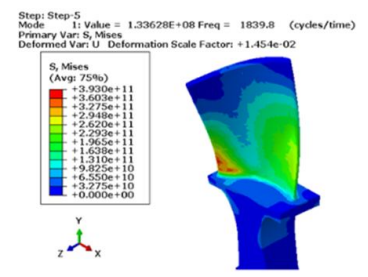

(a)

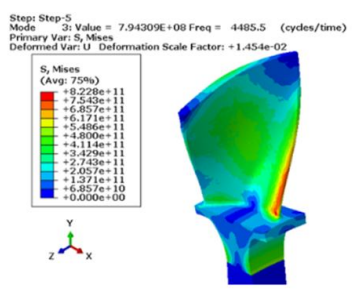

(c)

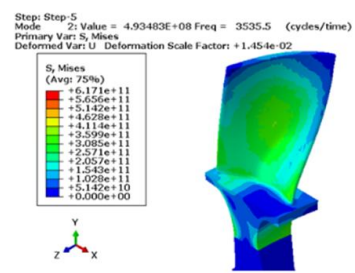

(b)

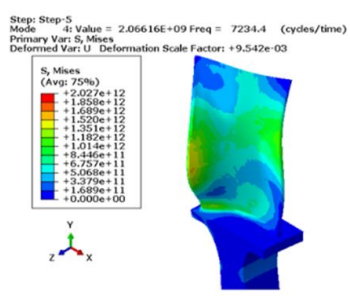

(d)

Fig.11. (a) first mode shape, (b) second mode shape, (c) third mode shape, and (d) fourth mode shape of blade. 
Table 3

The blade's first ten natural frequencies

\begin{tabular}{cccc}
\hline Mode number & Frequency $(\mathrm{Hz})$ & Mode number & Frequency $(\mathrm{Hz})$ \\
\hline 1 & 1840 & 6 & 13091 \\
2 & 3536 & 7 & 15570 \\
3 & 4486 & 8 & 16089 \\
4 & 7234 & 9 & 18971 \\
5 & 10690 & 10 & 19725 \\
\hline
\end{tabular}

Using Smith-Watson-Taper method a fatigue analysis of the blade based on modal analysis is performed via fe-safe software. The relation of this criterion is as follows:

$$
\sigma_{n-\max } \frac{\Delta \varepsilon_{n}}{2}=\frac{\sigma_{f}^{\prime 2}}{G}\left(2 N_{f}\right)^{2 b}+\sigma_{f}^{\prime} \varepsilon_{f}^{\prime}\left(2 N_{f}\right)^{b+c}
$$

This model is appropriate for materials which are damaged under tension mode (Han et al. 2002). Assuming maximum vertical stress on the critical plane causes the effect of mean stress to enter this criterion and since the maximum applied force on the blade is centrifugal force and the type of force is tensional, this criterion is used for blade analysis. According to Smith-Watson-Taper equation, the required parameters for the 718 super alloy are listed in Table 4.

Also, the surface quality coefficient is assumed to be $k_{t}=1$. Fig. 12 shows the blade's fatigue lifetime analysis contour, which is based on the modal analysis. According to this figure, the minimum blade lifetime is for 360000 cycle repeats. The connection region of the blade to the root is more vulnerable for cracking.

\section{Table 4}

Fatigue characteristics of 718 super alloy (Mitchell \& Landgraf, 1992).

\begin{tabular}{lcc}
\hline Const Amp Endurance limit (2nf) & $2 \mathrm{nf}$ & $1.00 \mathrm{E}+15$ \\
\hline Cyclic strength coefficient & $\mathrm{k}^{\prime}$ & 1564 \\
Cyclic strain hardening exponent & $\mathrm{n}^{\prime}$ & 0.0681 \\
\hline Fatigue strength exponent & $\mathrm{b}$ & -0.151 \\
Fatigue ductility exponent & $\mathrm{c}$ & -0.761 \\
Fatigue ductility coefficient & ef $^{\prime}$ & 1.5 \\
\hline Fatigue strength coefficient & Sf' or of $^{\prime}$ & 3950 \\
\hline
\end{tabular}

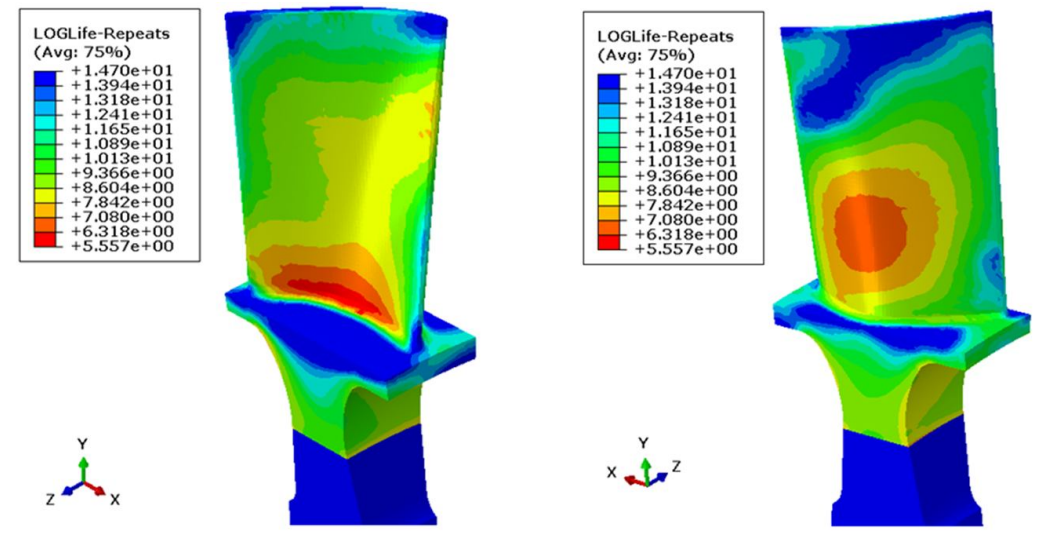

Fig. 12. Fatigue lifetime contour based on modal analysis 


\section{Calculating blade's lifetime within the interval of $\frac{2}{3} v_{\max }$ to $v_{\max }$}

In this section, assuming that the motor has two-thirds of its maximum possible revolutions at first and then velocity reaches its maximum by increasing the inlet temperature to $1000{ }^{\circ} \mathrm{C}$, blade's fatigue effect is analyzed. Accordingly, the maximum principal stresses are calculated via ABAQUS software. Fig. 13a and 13b shows the maximum principal stress for $20000 \mathrm{rpm}$ and $30000 \mathrm{rpm}$ revolutions, respectively.

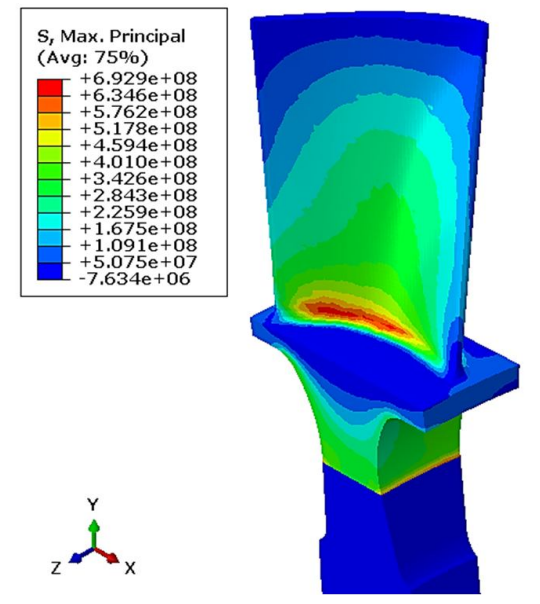

(a)

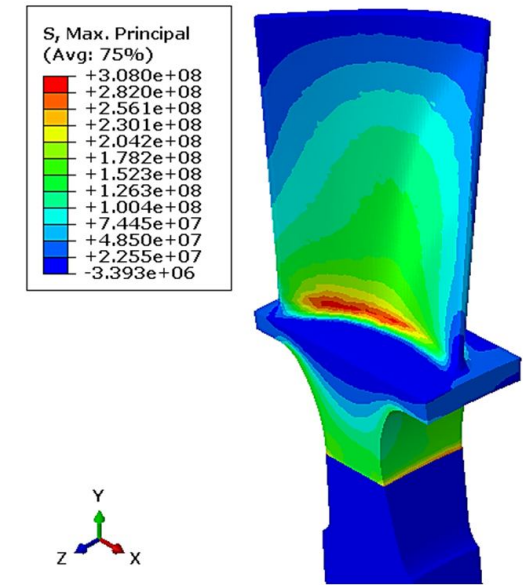

(b)

Fig. 13. maximum principal stress contour, a) maximum principal stress at $v_{\max }$ and b) maximum principal stress at $\frac{2}{3} v_{\max }$

As is shown from Fig. 14, $\sigma_{\text {min }}=308 M P a$ and $\sigma_{\text {max }}=692 \mathrm{MPa}$. Therefore, the fatigue loading diagram is as follows:

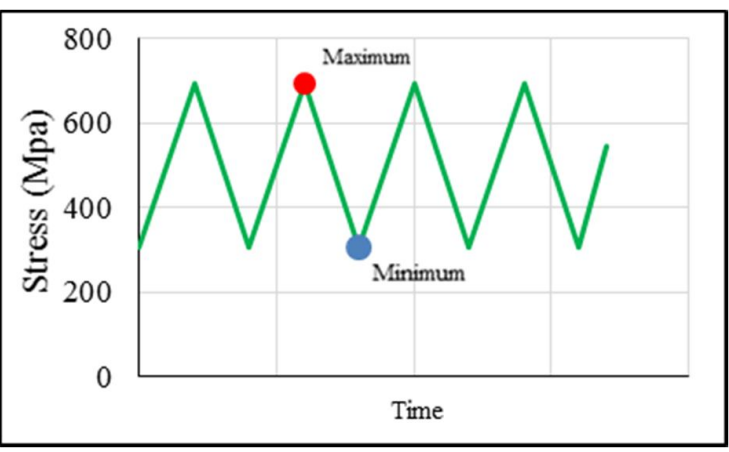

Fig.14. Fatigue loading diagram
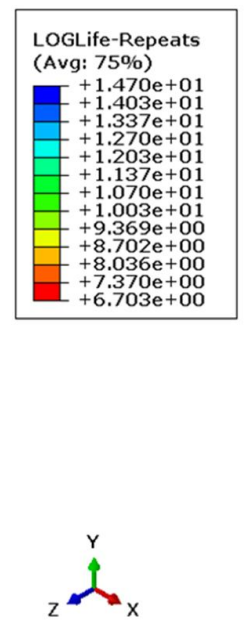

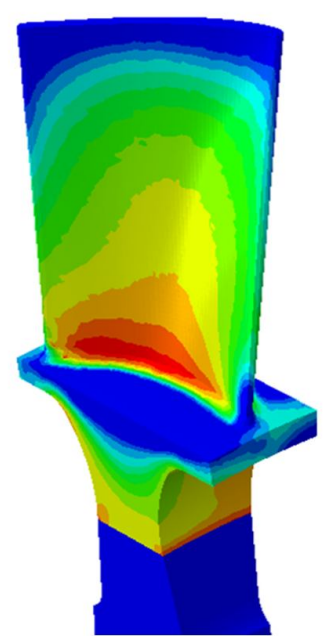

Fig. 15. Fatigue lifetime contour within the interval of $\frac{2}{3} v_{\max }$ to $v_{\max }$

As mentioned before, the analysis is performed at temperature of $1000{ }^{\circ} \mathrm{C}$. Fig. 15 shows the acquired results from this analysis. As shown in the figure, the blade's lifetime until the first crack appearance and failure is 5046612 cycle repeats. 


\section{Results and discussion}

Since the heat transfer is very high in super alloys and noting that micro turbine blades do not have a cooling mechanism, the temperature of the whole blade in spite of the presence of thermal coatings, quickly becomes similar with the combustion chamber temperature after 4.5 seconds. Therefore, no thermal stress is developed in these blades and only the mechanical characteristics of the super alloy vary as the temperature increases.

The worst case fatigue scenario which can occur for the turbine blades is that the blade is put under full cycle, so as the minimum stress $\left(\sigma_{\min }\right)$ reaches its minimum state and the maximum stress $\left(\sigma_{\max }\right)$ reaches its maximum state. In the previous sections, the full cycle loading was calculated by two loading methods. One of these loading methods is based on modal analysis, in which the fatigue analysis is performed by considering natural frequencies. According to Han et al. (2002) all the fatigue analyzes are performed based on Smith-Watson-Taper criterion, since this criterion is more appropriate for tension loads caused by centrifugal force comparing with other criteria. The acquired lifetime from the modal analysis and the other method are $10^{5.557}$ and $10^{5.868}$, respectively.

Also, according to Stephens et al.(2000), Fig. 16 shows the modified Maro and Goodman equations for specified long lifetimes (for instance, $10^{6}, 10^{7}$, and $10^{8}$ cycles). In the fatigue design process with constant amplitude loading and using parts with no slots, if the coordinates of the applied mean and periodic stresses $\left(\sigma_{m}\right.$ and $\sigma_{a}$, respectively) gets located between modified Maro or Goodman lines, which are shown in Fig.16, fatigue failure does not take place before designated lifetime. There is quite little difference between modified Maro and Goodman equations and both models often lead to similar results. However, if no yielding takes place, the applied mean and periodic stresses must be between two yield lines which connect $\pm S_{y}$ to $S_{y^{\prime}}$.

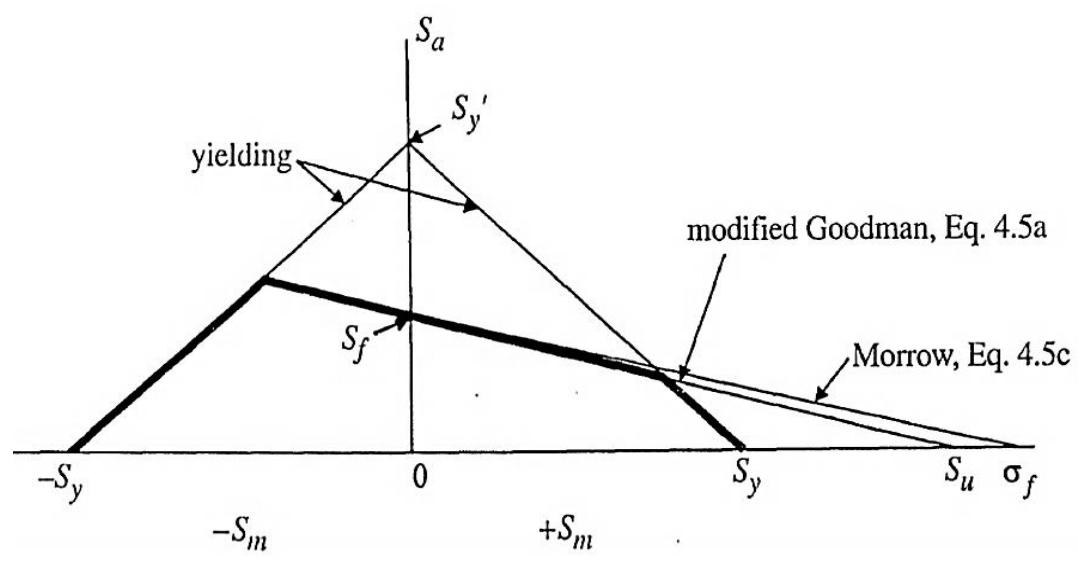

Fig. 16. Yield and fatigue criteria for constant lifetime parts with no slots (Stephens et al., 2000)

If neither fatigue nor yield failures happen, the stresses must violate none of the critical lines which are shown bold in Fig.16. Similarly, according to Figs. 10 and 14, which are relevant to full cycle loading and loading within the interval of $\frac{2}{3} v_{\max }$ to $v_{\max }$, we have:

$$
\begin{gathered}
\sigma_{m}=\frac{\sigma_{\text {max }}+\sigma_{\text {min }}}{2}=346.5 \mathrm{MPa} \\
\sigma_{m}=\frac{\sigma_{\text {max }}-\sigma_{\text {min }}}{2}=346.5 \mathrm{MPa}
\end{gathered}
$$

Within the interval of $\frac{2}{3} v_{\max }$ to $v_{\max }$ : 


$$
\begin{gathered}
\sigma_{m}=\frac{\sigma_{\text {max }}+\sigma_{\text {min }}}{2}=500 \mathrm{MPa} \\
\sigma_{m}=\frac{\sigma_{\text {max }}-\sigma_{\text {min }}}{2}=192 \mathrm{MPa}
\end{gathered}
$$

According to Mitchell \& Landgraf (1992), which is relevant to uniform stress-strain characteristics of 718 super alloy, the fatigue and yield criteria for 718 super alloy are depicted in Fig.17. As it is observed from the figure, the blade's lifetime when it is under full cycle loading is less than $10^{6}$ based on modified Maro and Goodman criterion and when the fatigue cycle is located within the interval of $\frac{2}{3} v_{\max }$ to $v_{\max }$, it can operate for at least $10^{6}$ cycles without any crack initiation based on Maro and Goodman criterion. This shows the accuracy and validity of finite element method in acquiring blade's fatigue lifetime.

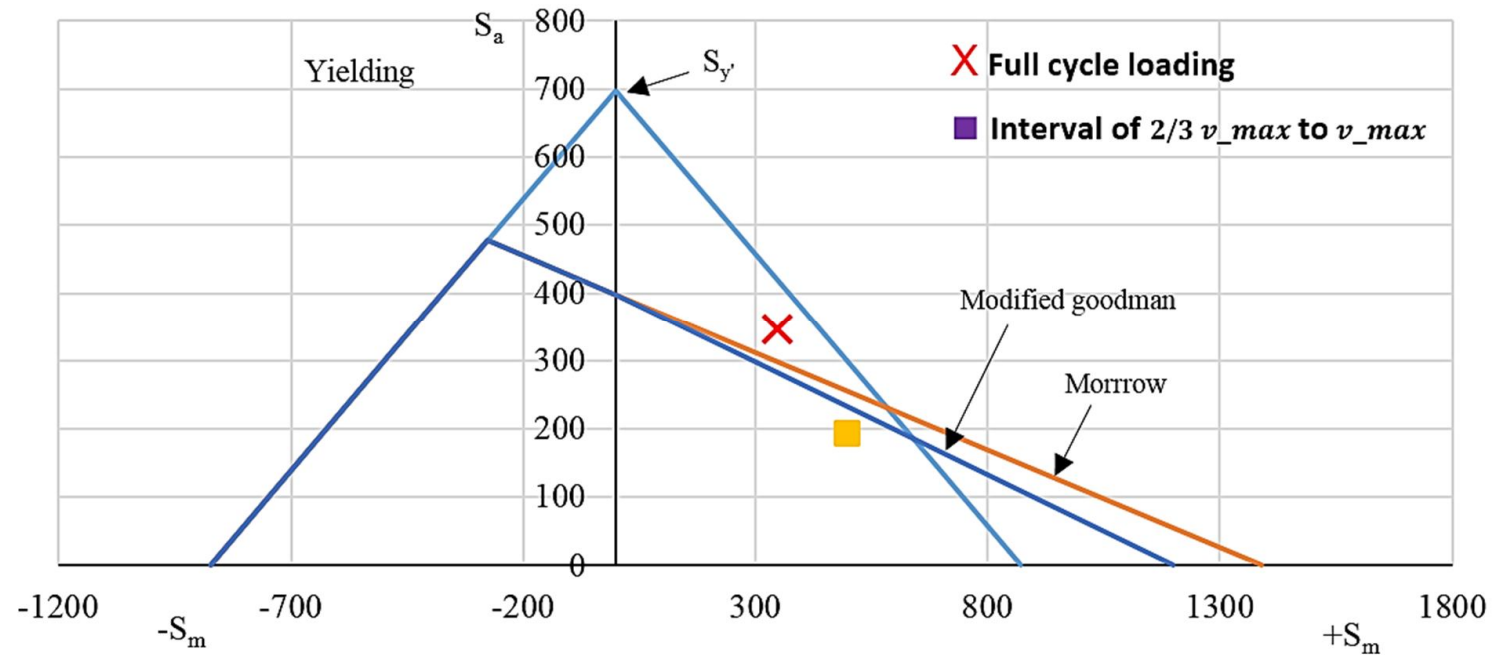

Fig. 17. Fatigue and yield criteria for 718 super alloy

Since the blade under centrifugal force effect remains in the elastic region, its lifetime can only be investigated from stress lifetime point of view and in addition to the above-mentioned validation, the Smith-Watson-Taper relation (i.e. Eq. 1) could be used for further validation. For example, for full cycle loading at $1000^{\circ} \mathrm{C}, E=109 \mathrm{GPa}, \Delta \varepsilon_{n}=0.0058$ and $\sigma_{n-\max }=692.9 \mathrm{Mpa}$ which by solving the above equation it is concluded that $\mathrm{N}=703475$. This number almost equals $10^{5.84}$ which is similar to finite element method results.

\section{Conclusions}

In this study, the fatigue effect of turbine's first blade row in TRI60 model turbofan motor is investigated and analyzed based on the transient modal analysis. For the purpose of estimating the fatigue and creeping lifetime of the turbine's first blade row, the heat transfer as well as thermal stress is analyzed for the blade via ABAQUS software. The results showed that in the lake of thermal coatings, the blade temperature becomes similar to the temperature of combustion chamber after 2 seconds and therefore, no stress due to temperature difference is developed in the blade. However, the blade's mechanical characteristics, namely, elasticity module and Poisson's ratio, vary as the temperature increases. Since the maximum revolution for the micro turbines equals $30000 \mathrm{rpm}$, the maximum stress due to centrifugal force takes place at the location of the connection of the blade and the disk.

According to Han et al.(2002), Smith-Watson-taper criterion was selected as the best criterion for fatigue analysis of the parts under tensile loads. After that, the fatigue and creeping lifetime of the blade 
was calculated based on the modal analysis with Smith-Watson-Taper criterion via fe-safe software. The fatigue lifetime of the blade was calculated under full cycle loading and loading within the interval of $\frac{\mathbf{2}}{\mathbf{3}} \boldsymbol{v}_{\max }$ to $\boldsymbol{v}_{\max }$ conditions. The acquired results were compared with yield and fatigue criteria for constant lifetime in parts with no slot (i.e. modified Goodman and Maro criteria). The acquired fatigue lifetime from finite element method for first and second loadings was $10^{5.5}$ and $10^{6.7}$, respectively and based on Maro and modified Goodman criterion, the blade's lifetime was less than $10^{6}$ when it is under

full cycle loading. Also, when the fatigue cycle is located within the interval of $\frac{\boldsymbol{2}}{\mathbf{3}} \boldsymbol{v}_{\max }$ to $\boldsymbol{v}_{\max }$, it can operate for at least $10^{6}$ cycles without any crack initiation based on Maro and Goodman criterion.

\section{References}

Chen, L. J., \& Xie, L. Y. (2005). Prediction of high-temperature low-cycle fatigue life of aeroengine's turbine blades at low-pressure stage. Journal of North Eastern University Natural Science, 26(7), 673.

Chen, L., Liu, Y., \& Xie, L. (2007). Power-exponent function model for low-cycle fatigue life prediction and its applications-Part II: Life prediction of turbine blades under creep-fatigue interaction. International Journal of Fatigue, 29(1), 10-19.

Han, C., Chen, X., \& Kim, K. S. (2002). Evaluation of multiaxial fatigue criteria under irregular loading. International Journal of Fatigue, 24(9), 913-922.

Kim, S. G., Hwang, Y. H., Kim, T. G., \& Shu, C. M. (2008). Failure analysis of J85 Engine turbine blades. Engineering Failure Analysis, 15(4), 394-400.

Metals, S. (2007). Inconel (R) alloy 718. Publication Number SMC-045. Special Metals Corporation.

Mitchell, M. R., \& Landgraf, R. W. (Eds.). (1992). Advances in fatigue lifetime predictive techniques (No. 1122). Astm International.

Salam, I., Tauqir, A., \& Khan, A. Q. (2002). Creep-fatigue failure of an aero engine turbine blades. Engineering Failure Analysis, 9(3), 335-347.

Stephens, R. I., Fatemi, A., Stephens, R. R., \& Fuchs, H. O. (2000). Metal fatigue in engineering. John Wiley \& Sons.

Wang, X., \& Xiao, P. (2004). Residual stresses and constrained sintering of YSZ/Al 2 O 3 composite coatings. Acta materialia, 52(9), 2591-2603.

Yan, X., Chen, X., Deng, Y., Sun, R., Lin, L., \& Nie, J. (2014). Investigation on Material's Fatigue Property Variation among Different Regions of DS Turbine Blades-Part A, Fatigue Tests on Full Scale Blades. Journal of Engineering for Gas Turbines and Power.

Yan, X., \& Nie, J. (2008). Creep-fatigue tests on full scale directionally solidified turbine blades. Journal of Engineering for Gas Turbines and Power, 130(4), 044501.

Yang, L., Liu, Q. X., Zhou, Y. C., Mao, W. G., \& Lu, C. (2014). Finite element simulation on thermal fatigue of a turbine blade with thermal barrier coatings. Journal of Materials Science \& Technology, 30(4), 371-380. 